Bridgewater State University

2016

\title{
Multi-frequency Ferromagnetic Resonance Investigation of Nickel Nanocubes Encapsulated in Diamagnetic Magnesium Oxide Matrix
}

Saritha Nellutla

Bridgewater State University, snellutla@bridgew.edu

Sudhakar Nori

North Carolina State University

Srinivasa R. Singamaneni

North Carolina State University

John T. Prater

U.S. Army Research Center

Jagdish Narayan

North Carolina State University

See next page for additional authors

Follow this and additional works at: http://vc.bridgew.edu/chem_fac

Part of the Chemistry Commons, and the Physics Commons

\section{Virtual Commons Citation}

Nellutla, Saritha; Nori, Sudhakar; Singamaneni, Srinivasa R.; Prater, John T.; Narayan, Jagdish; and Smirnov, Alix I. (2016). Multifrequency Ferromagnetic Resonance Investigation of Nickel Nanocubes Encapsulated in Diamagnetic Magnesium Oxide Matrix. In Chemical Sciences Faculty Publications. Paper 9.

Available at: http://vc.bridgew.edu/chem_fac/9 
Authors

Saritha Nellutla, Sudhakar Nori, Srinivasa R. Singamaneni, John T. Prater, Jagdish Narayan, and Alix I. Smirnov 


\title{
Multi-frequency ferromagnetic resonance investigation of nickel nanocubes encapsulated in diamagnetic magnesium oxide matrix
}

\author{
Saritha Nellutla, ${ }^{1, a)}$ Sudhakar Nori, ${ }^{2}$ Srinivasa R. Singamaneni, ${ }^{2,3, b)}$ John T. Prater, ${ }^{3}$ \\ Jagdish Narayan, ${ }^{2, a)}$ and Alex I. Smirnov ${ }^{4, a)}$ \\ ${ }^{1}$ Department of Chemical Sciences, Bridgewater State University, 24 Park Avenue, Bridgewater, \\ Massachusetts 02325, USA \\ ${ }^{2}$ NSF Center for Advanced Materials and Smart Structures, Department of Materials Science and Engineering, \\ North Carolina State University, 911 Partners Way, Raleigh, North Carolina 27695, USA \\ ${ }^{3}$ Materials Science Division, U.S. Army Research Office, P.O. Box 12211, Research Triangle Park, \\ North Carolina 27709, USA \\ ${ }^{4}$ Department of Chemistry, North Carolina State University, 2620 Yarbrough Drive, Raleigh, \\ North Carolina 27695, USA
}

(Received 22 August 2016; accepted 24 November 2016; published online 15 December 2016)

Partially aligned nickel nanocubes were grown epitaxially in a diamagnetic magnesium oxide ( $\mathrm{MgO}: \mathrm{Ni}$ ) host and studied by a continuous wave ferromagnetic resonance (FMR) spectroscopy at the X-band $(9.5 \mathrm{GHz})$ from $c a .117$ to $458 \mathrm{~K}$ and then at room temperature for multiple external magnetic fields/resonant frequencies from 9.5 to $330 \mathrm{GHz}$. In contrast to conventional magnetic susceptibility studies that provided data on the bulk magnetization, the FMR spectra revealed the presence of three different types of magnetic Ni nanocubes in the sample. Specifically, three different ferromagnetic resonances were observed in the X-band spectra: a line $\mathbf{1}$ assigned to large nickel nanocubes, a line $\mathbf{2}$ corresponding to the nanocubes exhibiting saturated magnetization even at ca. $0.3 \mathrm{~T}$ field, and a high field line $\mathbf{3}$ ( $g_{\text {eff }} \sim 6.2$ ) tentatively assigned to small nickel nanocubes likely having their hard magnetization axis aligned along or close to the direction of the external magnetic field. Based on the analysis of FMR data, the latter nanocubes possess an anisotropic internal magnetic field of at least $\sim 1.0 \mathrm{~T}$ in magnitude. Published by AIP Publishing.

[http://dx.doi.org/10.1063/1.4971869]

\section{INTRODUCTION}

Ferromagnetic nanoparticles exhibit many interesting properties including single magnetic domain behavior when their diameters do not exceed $c a$. $50 \mathrm{~nm}$. The single domain nanostructures are viewed as promising candidates for nanodevices including high-density nonvolatile memory technology. Specifically, by arranging such nanoparticles as planar periodic arrays, one would envision an attractive alternative to traditional multilayer magnetic media employed in the magnetic hard disks. ${ }^{1,2}$ Generally, nanoparticle arrays can be formed by patterning including lithography or by either self-assembly in diamagnetic matrices ${ }^{3}$ or epitaxial growth on crystalline media. ${ }^{4}$ Self-assembly within the thin film semiconductors is a particularly promising technology for producing uniform-size, defect-free, and, in several cases, ordered nanostructures whose feature size is well below the current lithographic limit. ${ }^{3}$ As the fabrication methods for nanoparticle arrays by both patterning and self-assembly are progressing further and further, it is imperative to understand the underlying spin physics in such precipitate/nanodot systems.

Nickel is one of the only four elements that are magnetic at or near room temperature with others being iron, cobalt,

\footnotetext{
a) Authors to whom correspondence should be addressed. Electronic addresses: snellutla@bridgew.edu, Telephone: +1 5085313946; jay_narayan@ ncsu.edu, Telephone: +1 9195157874; and alex_smirnov@ncsu.edu, Telephone: +19195134377.

${ }^{b)}$ Current address: Department of Physics, University of Texas at El Paso, El Paso, Texas 79968, USA.
}

and gadolinium. It is widely available and has a good chemical stability due to the formation of an oxide layer on the surface. Thus, it is a very attractive metal for fabrication of ferromagnetic nanoparticles and arrays in a variety of host materials such as diamagnetic magnesium oxide $(\mathrm{MgO})$. The latter is advantageous for the following reasons: $\mathrm{Mg}$ is an abundantly available element in the Earth's crust and $\mathrm{MgO}$ is known to readily form cubic crystals that can be grown fairly "defect-free" from the melt.

In the past, Narayan et al. described the synthesis and characterization of partially ordered Ni nanoparticles embedded in a $\mathrm{MgO}$ matrix (here onwards, $\mathrm{MgO}: \mathrm{Ni}$ ). ${ }^{4,5}$ Conventional and high resolution TEM images (e.g., Fig. 1(a)) of MgO:Ni revealed cube-shaped Ni nanocrystals with sizes ranging from $c a .10$ to $100 \mathrm{~nm}$ and an average edge length of $c a .50 \mathrm{~nm}$. Further, the bases of the Ni nanocubes were epitaxially oriented along the orthogonal $\langle 110\rangle_{\mathrm{MgO}}$ directions. ${ }^{4,5}$ Electron and neutron diffraction studies indicated that along any given $\langle 100\rangle_{\mathrm{MgO}}$ direction, there are four different orientations of $\mathrm{Ni}$ nanocubes, namely, $\langle 11 \overline{1}\rangle_{\mathrm{Ni}}\left\|\langle 100\rangle_{\mathrm{MgO},}\langle 1 \overline{1} 0\rangle_{\mathrm{Ni}}\right\|\langle 100\rangle_{\mathrm{MgO}},\langle 112\rangle_{\mathrm{Ni}} \|$ $\langle 100\rangle_{\mathrm{MgO}}$, and $\langle 100\rangle_{\mathrm{Ni}} \|\langle 100\rangle_{\mathrm{MgO}}{ }^{4}$. All the orientations are about equally distributed; however, a long annealing at high temperatures was found to enhance the $\langle 100\rangle_{\mathrm{Ni}} \|\langle 100\rangle_{\mathrm{MgO}}$ orientation. ${ }^{4} \mathrm{~A}$ schematic representation of the sample crystallographic directions is shown in Figure 1(b). It is worth noting here that even though $\mathrm{MgO}: \mathrm{Ni}$ samples were originally synthesized nearly a decade or so ago, these composite nanostructures are very robust based on TEM/SEM experiments and consistent correlations with optical, electrical, and magnetic 


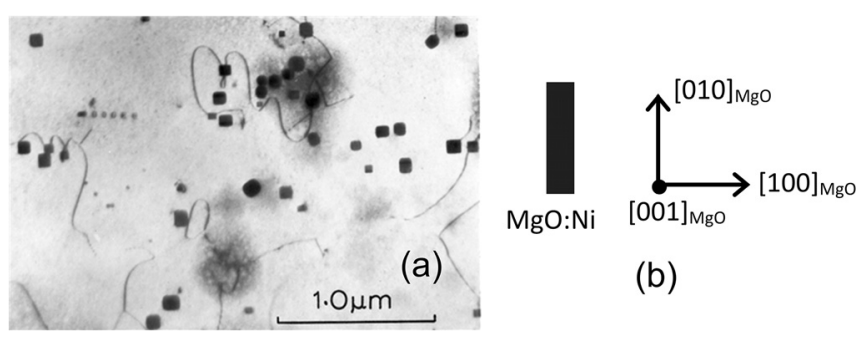

FIG. 1. (a) A representative transmission electron (TEM) micrograph illustrating a macroscopic alignment of $\mathrm{Ni}$ nanocubes in the $\mathrm{MgO}$ matrix. The viewing direction is along the $[001]_{\mathrm{MgO}}$ crystal axis. (b) Schematic representation of the MgO:Ni crystal and its crystallographic directions.

properties carried out over the years. This exceptionally high stability of the samples is attributed to extremely low diffusivities of the intrinsic point defects, vacancies in particular, with an activation barrier of $\sim 4.0 \mathrm{eV}^{6}$

Previous variable temperature DC magnetic susceptibility studies $^{5,7}$ revealed that under an applied magnetic field of 0.05 T, MgO:Ni exhibits superparamagnetism with the blocking temperature above room temperature. ${ }^{5,7}$ As expected, a difference between magnetization $v s$. temperature curves measured for the zero-field and field-cooled samples vanished when the applied magnetic field was increased to $1 \mathrm{~T}^{5,7}$ Further, the variable temperature magnetization studies of $\mathrm{MgO}: \mathrm{Ni}$ from 5 to $350 \mathrm{~K}$ revealed changes in the coercive field from 31.5 to $0.6 \mathrm{mT}$ and in the saturation field from 570 to $375 \mathrm{mT}$, respectively. These observations suggest that $\mathrm{MgO}: \mathrm{Ni}$ exhibits a strong bulk ferromagnetism below $\mathrm{ca}$. $300 \mathrm{~K}^{7}$

Once the bulk ferromagnetic behavior of $\mathrm{Ni}$ nanocubes in a diamagnetic $\mathrm{MgO}$ host has been established by the $\mathrm{DC}$ magnetometry, it is imperative to further characterize the spin exchange and local anisotropy field phenomena in such macroscopically organized anisotropic nanoscale system. Thus, we turned to ferromagnetic resonance (FMR) as a spectroscopic tool to differentiate and characterize the magnetic interactions originating from various components of $\mathrm{MgO}: \mathrm{Ni}$ as well as to evaluate the strength of the local magnetic anisotropy fields in Ni nanocubes. FMR is also an informative technique to investigate the internal spin dynamics responsible for the relaxation processes in ferromagnetic nanoparticle systems. ${ }^{8-15}$ Here, we describe the FMR studies of $\mathrm{MgO}: \mathrm{Ni}$ to gain a physical insight into magnetic interactions and local anisotropy field phenomena.

\section{EXPERIMENT}

The continuous wave (CW) X-band $(9.5 \mathrm{GHz})$ FMR spectra were recorded as a function of crystal orientation and temperature using the Elexsys 580 spectrometer equipped with a super high Q cavity and a variable temperature with continuous flow variable temperature system capable of maintaining temperature from 117 to $458 \mathrm{~K}$ (all from Bruker Biospin, Karlsruhe, Germany). All the measurements were carried out in $\mathrm{N}_{2}$ atmosphere in order to prevent any interference from the paramagnetic molecular oxygen. The best signal-to-noise ratio for FMR spectra with essentially no line shape distortions was obtained when the incident microwave power was at $1.002 \mathrm{~mW}$ and the amplitude of $100 \mathrm{kHz}$ magnetic field modulation was at $1 \mathrm{mT}$.

Multi-frequency FMR spectra from 24 to $330 \mathrm{GHz}$ were measured at room temperature ( $c a .295 \mathrm{~K}$ ) using spectrometers developed at the National High Magnetic Field Laboratory in Tallahassee, FL. The K-band (24 GHz) FMR measurements were conducted on a Physical Property Measurement System (PPMS, Quantum Design, San Diego, CA) using a split-coil transverse $7 \mathrm{~T}$ magnet. Microwave frequency was generated and detected using a solid state millimeter-wave vector network analyzer (AB Millimetre, Paris, France). ${ }^{16}$ FMR spectra were collected in the absorption mode using a cylindrical resonator operating in one of the $\mathrm{TE}_{01 \mathrm{n}}$ modes. ${ }^{15}$ FMR spectra at a series of resonant frequencies from 50 to $330 \mathrm{GHz}$ were measured using a transmission spectrometer equipped with a $17 \mathrm{~T}$ superconducting magnet and a solid state microwave source with multiple multiplication steps. ${ }^{17}$ A quasi-optical EPR spectrometer with a heterodyne detection and a $12.5 \mathrm{~T}$ superconducting magnet was employed for FMR at $240 \mathrm{GHz} .{ }^{18}$

All the FMR spectra were least-squares fit to a superposition of three pseudo-Voigt lineshape functions (i.e., sums of Gaussian and Lorentzian functions) using an EPRCalc program. ${ }^{19}$ During the fitting, the field positions, intensities, and the widths of Gaussian and Lorentzian components were adjusted independently for each of the three lines. The goodness of all the fits was within $\pm 5 \%$ of the signal amplitude.

\section{RESULTS AND DISCUSSION}

Each ferromagnetic nanoparticle is expected to possess an internal magnetic field, $\overrightarrow{b_{\text {int }}}$, arising from various anisotropic contributions such as magnetocrystalline $\left(K_{1}\right)$, shape, surface, spin-exchange, and strain fields. ${ }^{20}$ In the absence of an external magnetic field, $\overrightarrow{B_{\text {ext }}}$, the thermodynamic equilibrium orientation of the ferromagnetic nanoparticle magnetic moment, $\mu_{\mathrm{NP}}$, is along $\overrightarrow{b_{\mathrm{int}}}$ and the ferromagnetic resonance (FMR) condition is given by $h \nu=\beta \cdot \boldsymbol{g}_{\text {eff }} \cdot \overrightarrow{b_{\text {int }}}$, where $h$ is the Planck constant, $\nu$ is the microwave frequency, $\boldsymbol{g}_{\text {eff }}$ is the effective $g$-tensor, and $\beta$ is the electron Bohr magneton. In an external field $\overrightarrow{B_{\text {ext }}}$, the FMR condition changes to $h \nu$ $=\beta \cdot g_{\text {eff }} \cdot \overrightarrow{b_{\text {eff }}}$, where the effective field $\overrightarrow{b_{\text {eff }}}=\overrightarrow{B_{\text {ext }}}+\overrightarrow{b_{\text {int }}}$. Further, for a system with a broad particle size distribution, like the MgO:Ni arrays studied here, the following modifications to the effective resonance conditions should be made:

(i) The total effective magnetic moment is given by $\overrightarrow{\mu_{\text {eff }}}=\sum \overrightarrow{\mu_{\mathrm{NP}}}$, where the summation is over all the nanocubes.

(ii) The total internal field generated is $\overrightarrow{B_{\text {int }}}=\sum \overrightarrow{b_{\text {dipole }}}$ $+\sum \overrightarrow{b_{\text {int }}}$, where $\sum \overrightarrow{b_{\text {int }}}$ reflects the summation over all nanocubes and $\sum \overrightarrow{b_{\text {dipole }}}$ corresponds to the summation of all the inter-cube dipolar interactions.

(iii) In the presence of an external magnetic field, the system experiences an effective magnetic field $\overrightarrow{B_{\text {eff }}}$ $=\overrightarrow{B_{\text {ext }}}+\overrightarrow{B_{\text {int }}}$ and the resonance condition is given as $h \nu=\beta \cdot \boldsymbol{g}_{\text {eff }} \cdot \overrightarrow{B_{\text {eff }}}$. Therefore, the FMR resonance line position depends not only on the relative strength of $\left|B_{\text {ext }}\right|$ and $\left|B_{\text {int }}\right|$ but also on their relative orientation. 
Further, a large FMR linewidth is expected because of the overlapping resonance signals from the individual nanocubes at different resonance conditions.

\section{A. Room temperature X-band FMR spectrum}

The room temperature X-band $(9.5 \mathrm{GHz})$ FMR spectrum of the MgO:Ni crystal ( $c f$. Fig. 2) exhibits several broad resonance lines spread over the entire range of the magnetic field (i.e., from ca. 0 to $1.4 \mathrm{~T}$ ) accessible with the largest Bruker Biospin electromagnet E073 without employing supplementary pole caps. An additional pattern of six sharp lines centered at $g \approx 2(\sim 0.34 \mathrm{~T})$ and almost equally split by $\sim 9 \mathrm{mT}$ was attributed to $\mathrm{Mn}^{2+}$ impurities (at $c a$. $\mu \mathrm{M}$ level) in the MgO:Ni crystal. The $\mathrm{Mn}^{2+}$ EPR signal did not interfere with the FMR data interpretation.

Large linewidths are consistent with the broad size distribution of $\mathrm{Ni}$ nanocubes observed in TEM and magnetic susceptibility data. ${ }^{5,7}$ However, an observation of the three FMR lines (referred to as $\mathbf{1}, \mathbf{2}$, and $\mathbf{3}$ from here on) with some of the lines only partially resolved at certain orientations is somewhat surprising, especially since nickel is considered to be a soft ferromagnet. If the magnitude of the Zeeman field $\left|\overrightarrow{B_{\text {ext }}}\right|$ is larger than the net saturation magnetic field of $\mathrm{MgO}: \mathrm{Ni}$, one expects to observe a single broad "superparamagnetic" line centered at $B_{\text {res }}^{0}=\frac{h \nu}{g_{0} \beta}=0.307 \mathrm{~T}$, where $\nu=9.50 \mathrm{GHz}$ and $g_{0}=g_{\mathrm{Ni}}=2.20$. The presence of the resonance lines $\mathbf{1}$ and $\mathbf{3}$ in the MgO:Ni FMR spectrum indicates the presence of $\mathrm{Ni}$ nanocubes with internal magnetic fields much larger than the Zeeman field accessible with the $\mathrm{X}$-band EPR electromagnet (i.e., up to $1.4 \mathrm{~T}$ ). Therefore, the three resonance lines are assigned as follows, assuming that the effective magnetic moments of each of the nanocubes coincide with its $\overrightarrow{b_{\text {eff }}}$ direction:

The resonance line $\mathbf{1}$ arises from Ni nanocubes exhibiting "bulk" properties, most likely the large multidomain nanocubes. $B_{\text {res }}(\mathbf{1})<B_{\text {res }}{ }^{0}$ implies not only that the magnitude of the net internal magnetic field

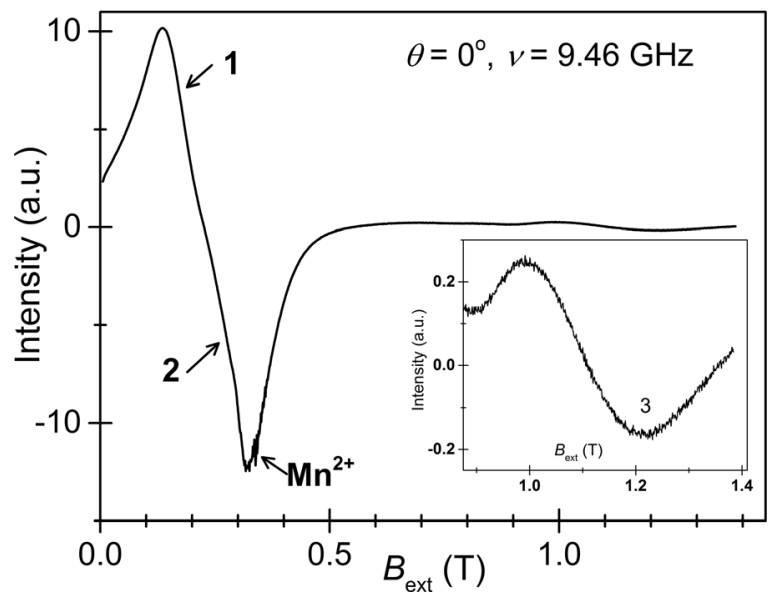

FIG. 2. (a) The X-band (9.5 GHz) FMR spectrum of MgO:Ni at room temperature for $B_{\text {ext }}$ aligned along the $[100]_{\mathrm{MgO}}$ crystal direction, i.e., $\theta \sim 0^{\circ}$. 1, 2, and 3 correspond to three sets of Ni nanocubes. See text for further details. The inset shows $c a$. tenfold magnified high field portion of the FMR spectrum. of these Ni nanocubes, $B_{\text {int }}(\mathbf{1})$, is significant in comparison to the Zeeman field but also that its direction is along $\overrightarrow{B_{\text {ext }}}$.

(ii) Since the observed $B_{\text {res }}(2)$ is close to $B_{\text {res }}{ }^{0}$, the effect of the net internal field, $B_{\text {int }}(2)$, on $B_{\text {res }}(2)$ is minimal and the Zeeman field must be sufficient to align the magnetic moments of set $\mathbf{2} \mathrm{Ni}$ nanocubes along the applied field direction.

(iii) FMR line $\mathbf{3}$ arises also from unsaturated Ni nanocubes, but $B_{\text {res }}(3) \ggg B_{\text {res }}{ }^{0}$ implies that these Ni nanocubes have a very large net internal magnetic field $B_{\text {int }}(3)$ that is not aligned along the $\overrightarrow{B_{\text {ext }}}$ direction. We speculate that these $\mathrm{Ni}$ nanocubes are small and that their surface spins exhibit significantly different properties vs. the core spins.

\section{B. Temperature dependence of the X-band FMR spectra}

Initially, the FMR spectra of the MgO:Ni crystal were measured from 117 to $458 \mathrm{~K}$ at $9.5 \mathrm{GHz}$ (X-band) with $\overrightarrow{B_{\text {ext }}}$ directed along the $[100]_{\mathrm{MgO}},[010]_{\mathrm{MgO}}$, and $[001]_{\mathrm{MgO}}$ crystal axes. As expected for a cubic crystal, the temperature dependence of the FMR spectrum is identical for all the three directions (data not shown). However, the very high field portion of the resonance line $\mathbf{3}$ could not be entirely collected at low temperatures due to the limitations of the maximum magnetic field provided by the Bruker electromagnet $\left(B_{\mathrm{ext} \text { max }} \approx 1.4 \mathrm{~T}\right)$. Therefore, in order to retain the resonance line $\mathbf{3}$ within the magnetic field range accessible with the Bruker electromagnet, the temperature dependence of the FMR spectrum was collected for $\theta \sim 6^{\circ}$, where $\theta$ is the angle between $\overrightarrow{B_{\text {ext }}}$ and the $[100]_{\mathrm{MgO}}$ crystal direction with $B_{\text {ext }}$ in the $\{010\}$ plane (see Fig. 1(b)).

Figure 3 shows the FMR spectra of $\mathrm{MgO}: \mathrm{Ni}$ at representative temperatures. As the temperature increases from $117 \mathrm{~K}$ to $458 \mathrm{~K}$, the resonance lines $\mathbf{1}$ and $\mathbf{2}$ appear to narrow and shift towards $B_{\text {res }}{ }^{0}$ ( $c f$. Fig. 3(a)). Unfortunately, for $T<250 \mathrm{~K}$, the resonance lines from the sets $\mathbf{1}$ and $\mathbf{2}$ were merging together, and therefore, the low-field resonance lines below $250 \mathrm{~K}$ could not be assigned to either set 1 or set $2 \mathrm{Ni}$ nanocubes with any degree of certainty. Hence, for $\mathbf{1}$ and 2 lines, the effect of temperature on $B_{\text {res }}$ and linewidth $\delta B$ (defined as the full width at half maximum, FWHM) will be discussed only within the $250-458 \mathrm{~K}$ range. For the resonance line 3, both the position and the FWHM remain nearly unchanged from $117 \mathrm{~K}$ to $250 \mathrm{~K}$ ( $c f$. Fig. 3(b)), but the signal narrowed and its field position shifted towards $B_{\text {res }}{ }^{0}$ for $T>250 \mathrm{~K}$.

All three resonance lines $(\mathbf{1}, \mathbf{2}, \mathbf{3})$ were simultaneously least-square fitted to a superposition of pseudo-Voigt functions with the amplitude, $B_{\text {res }}, \delta B$, and a lineshape contribution factor, $\alpha$, as adjustable parameters. The parameter $\alpha$ changes from $\alpha=0$ for a pure Gaussian lineshape to $\alpha=1$ for a pure Lorentzian function. ${ }^{19}$ This fitting model describes the X-band FMR spectra at different temperatures reasonably well with all the fit residuals, i.e., the differences between the simulated and the experimental spectra, $\leq \pm 5 \%$ of the amplitudes of the experimental spectra ( $c f$. Fig. 4). 

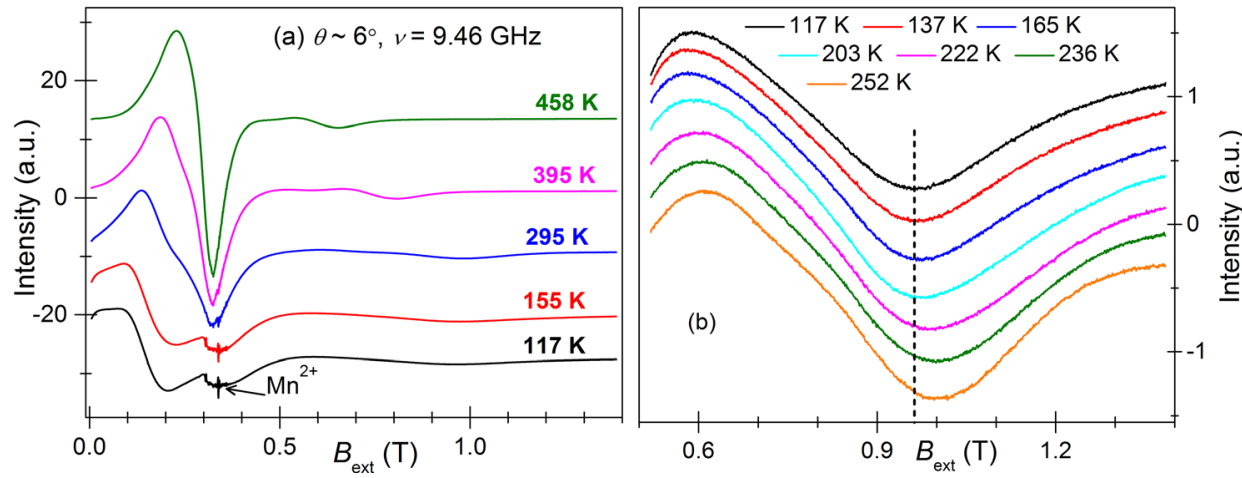

FIG. 3. (a) Representative variable temperature X-band (9.5 GHz) FMR spectra of MgO:Ni at $\theta \sim 6^{\circ}$. $B_{\text {ext }}$ is in the $\{010\}_{\mathrm{MgO}}$ plane with $\theta$ as the angle between $B_{\text {ext }}$ and the $[100]_{\mathrm{MgO}}$ crystal direction (cf. Fig. 1(b)). (b) A series of magnified FMR lines corresponding to set 3 Ni nanocubes from 117 to $252 \mathrm{~K}$. The vertical dotted line is a guide for an eye to demonstrate the nearly unchanged $B_{\text {res }}$ of set $3 \mathrm{Ni}$ nanocubes below $\sim 200 \mathrm{~K}$. The spectra in (a) and (b) are shifted vertically for clarity.

Figure 5 summarizes the temperature dependencies of $B_{\text {res }}$, and $\delta B$ of the resonance lines 1 and 2 from $250 \mathrm{~K}$ to $458 \mathrm{~K}$ and the resonance line $\mathbf{3}$ from $117 \mathrm{~K}$ to $458 \mathrm{~K}$. As the temperature increases, $B_{\text {res }}(1)$ gradually increases from $\sim 0.18 \mathrm{~T}$ (at $250 \mathrm{~K}$ ) to $\sim 0.26 \mathrm{~T}$ (at $458 \mathrm{~K}$ ), while $B_{\text {res }}(2)$ weakly increases (i.e., $\sim 0.28$ to $\sim 0.30 \mathrm{~T}, c f$. Fig. 5(a)) within the same temperature range.

The FMR signal linewidth of set $\mathbf{1}(\delta B(\mathbf{1}))$ and set $\mathbf{2}$ $(\delta B(\mathbf{2}))$ nickel nanocubes decreases almost linearly over the entire range. These are expected as the thermal fluctuations of the net magnetic moment $\mu_{\mathrm{eff}}(i)(i=\mathbf{1}, \mathbf{2})$ around its equilibrium orientation increase with temperature, leading to some averaging of the effects of the net internal fields on $B_{\text {res }}$ and $\delta B$. Hence, both $B_{\text {res }}(\mathbf{1})$ and $B_{\text {res }}(\mathbf{2})$ shift towards the "superparamagnetic" resonance limit for Ni which is $\sim 0.31 \mathrm{~T}$ for $v \sim 9.5 \mathrm{GHz}$. Neglecting the effect of the crystal rotation by $\theta=6^{\circ}$, non-coincidence of the $B_{\text {res }}(\mathbf{1})$ with that of the $B_{\text {res }}(\mathbf{2})$ at $458 \mathrm{~K}$ suggests that even at this temperature, the thermal energy is not sufficient to completely average out the internal magnetic field of the set $\mathbf{1} \mathrm{Ni}$ nanocubes in MgO:Ni. Further, a relatively large difference between the linewidths of the set $\mathbf{1}$ $(\sim 130 \mathrm{mT})$ and the set $\mathbf{2}(\sim 60 \mathrm{mT})$ of $\mathrm{Ni}$ nanocubes reflects their respective distributions of sizes in $\mathrm{MgO}: \mathrm{Ni}$ with the set $\mathbf{1}$ having broader distributions $v s$. the set $\mathbf{2}$.

$B_{\text {res }}(\mathbf{3})$ vs. $T$ plot ( $c f$. Fig. 5(c)) exhibits a maximum at ca. $236 \mathrm{~K}-268 \mathrm{~K}$ followed by a pseudo-plateau at $\sim 0.9 \mathrm{~T}$ in the $117 \mathrm{~K}-211 \mathrm{~K}$ range. The FMR signal linewidth of set 3 nickel nanocubes, $\delta B(\mathbf{3})$, gradually increases from $\sim 180 \mathrm{mT}$ to $\sim 260 \mathrm{mT}$ as the temperature decreases from $458 \mathrm{~K}$ to $117 \mathrm{~K}$ and exhibits a plateau below $\sim 200 \mathrm{~K}$. We note that since the position of the resonance line $\mathbf{3}$ is not shifted below $\sim 200 \mathrm{~K}$ (cf. Fig. 3(b)), within the available spectral resolution, the observed increase in $B_{\text {res }}(3)$ from $117 \mathrm{~K}$ to $200 \mathrm{~K}$ could be either an artifact of the least-squares fitting of the overlapping components or caused by a slight dislocation of the sample and/or the sample rod. Considering an exceptionally broad width of line 3 ( $c f$. Fig. 3(b)) at low temperatures, it is not very surprising to see such an artifact in the values of $B_{\text {res }}$ obtained by the least-squares fitting, especially for a simplified model. Similar to the set $\mathbf{1} \mathrm{Ni}$ nanocubes, $B_{\text {res }}(3)$ reaches only $\sim 0.6 \mathrm{~T}$ at $\sim 460 \mathrm{~K}$ instead of the expected "superparamagnetic limit" of $\sim 0.31 \mathrm{~T}$ (for $v \sim 9.5 \mathrm{GHz}$ ). This implies that the anisotropic interactions producing a net internal magnetic field in the set $3 \mathrm{Ni}$ nanocubes persist up to $\sim 460 \mathrm{~K}$.

As discussed at the beginning of this section, for a ferromagnetic nanoparticle, a difference between the experimentally observed resonance position $\left(B_{\text {res }}\right)$ and its "superparamagnetic" resonance position $\left(B_{\mathrm{res}}{ }^{0}\right)$ is associated with its net internal field $\left(B_{\text {int }}\right)$. Further, this difference is proportional to the magnetization of the ferromagnetic nanoparticle. Since only the nanocube sets $\mathbf{1}$ and $\mathbf{3}$ show considerable shifts of the resonance position from $B_{\text {res }}{ }^{0},\left|B_{\text {res }}-B_{\text {res }}{ }^{0}\right|$ is plotted as a function of
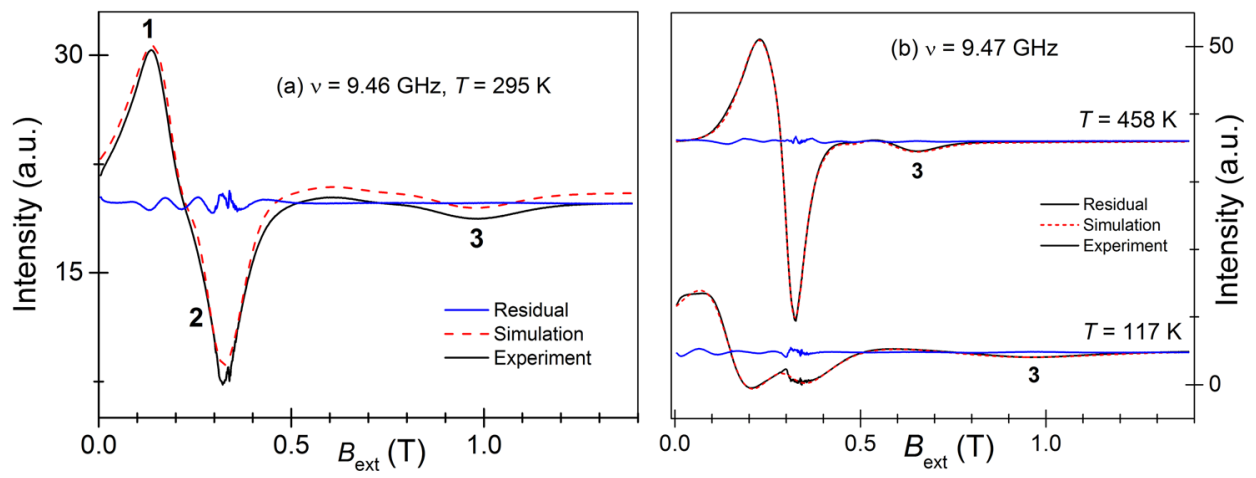

FIG. 4. (a) Experimental (black solid line) and the least-square fitted (red dashed line) $295 \mathrm{~K} \mathrm{X}$-band (9.5 GHz) FMR spectrum of MgO:Ni at $\theta \sim 6^{\circ}$, where $\theta$ is the angle between $B_{\text {ext }}$ and the [100 $]_{\mathrm{MgO}}$ crystal direction with $B_{\mathrm{ext}}$ in the $\{010\}_{\mathrm{MgO}}$ plane. (b) Simulated and experimental X-band FMR spectra at $117 \mathrm{~K}$ and $458 \mathrm{~K}$ for $\theta \sim 6^{\circ}$. The fit residuals—-the differences between the experimental and simulated spectra-are shown as blue lines. The spectra in (a) and (b) are vertically shifted for clarity. 

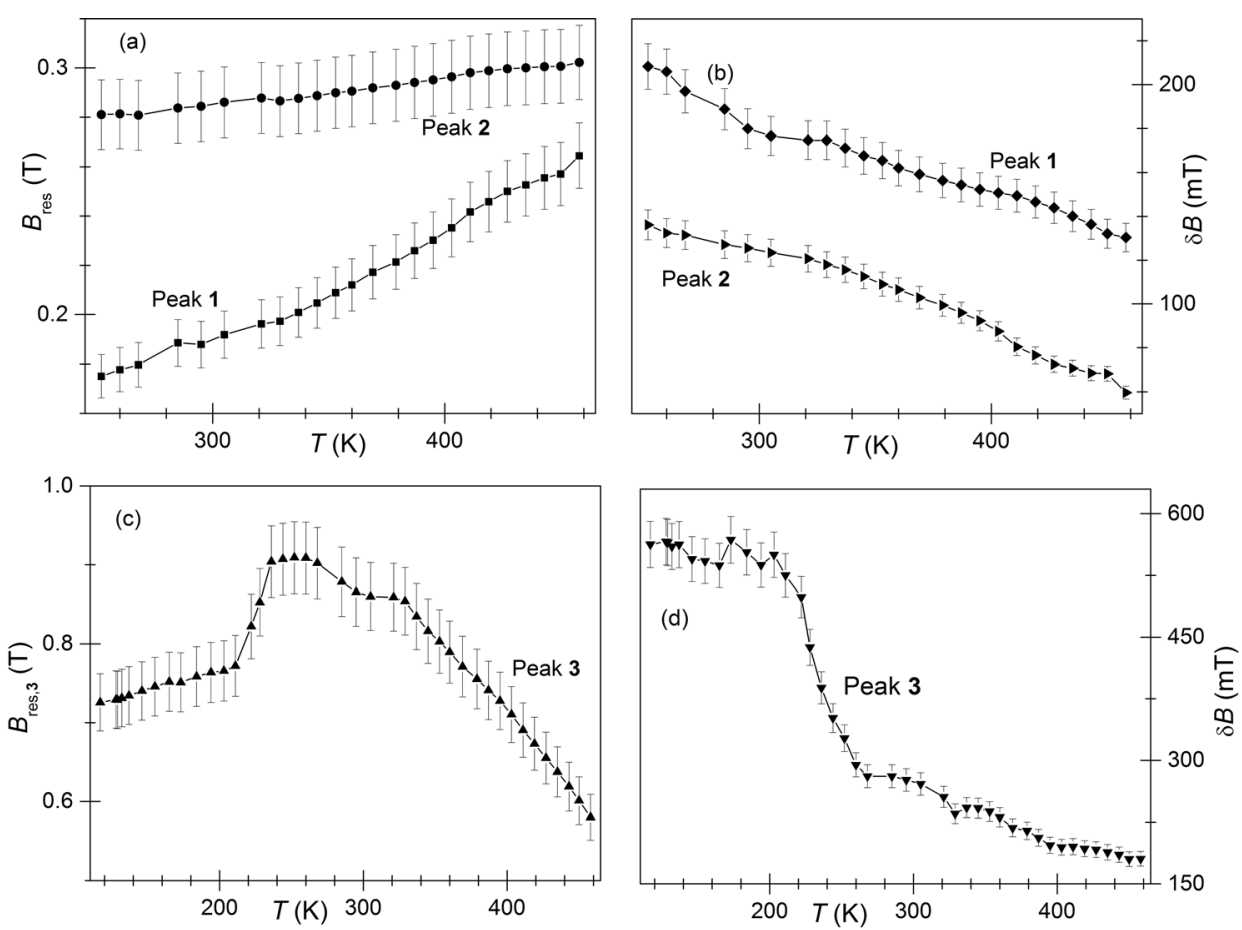

FIG. 5. Temperature dependence of $B_{\text {res }}$ and $\delta B$ of the X-band $(9.5 \mathrm{GHz})$ FMR lines 1, 2, and 3 of $\mathrm{MgO}: \mathrm{Ni}$ at $\theta \sim 6^{\circ}$, where $\theta$ is the angle between $B_{\text {ext }}$ and the $[100]_{\mathrm{MgO}}$ crystal direction with $B_{\text {ext }}$ in the $\{010\}_{\mathrm{MgO}}$ plane. The solid lines connecting the experimental data points are given as a guide to the eye. temperature in Fig. 6 for these two sets of nanocubes. Even though $\left(B_{\text {res }}{ }^{0}-B_{\text {res }}, 1\right)$ parameter for the set 1 Ni nanocubes cannot be obtained below $c a$. $240 \mathrm{~K}$, its decrease with an increase in temperature ( $c f$. Fig. 6(a)) reflects the expected high temperature behavior of magnetization $(M)$ for a ferromagnetic nanoparticle system. Interestingly, $\left(B_{\text {res }}-B_{\text {res }}{ }^{0}\right)$ vs. $T$ data for set $3 \mathrm{Ni}$ nanocubes is very reminiscent of a typical temperature dependence of the saturation magnetization for a ferromagnetic system. ${ }^{21,22}$ This, in combination with the fact that $B_{\text {res }}(3)$ is much greater than $B_{\text {res }}{ }^{0}$ in the temperature range studied, implies that the set $3 \mathrm{Ni}$ nanocubes in $\mathrm{MgO}: \mathrm{Ni}$ has a sufficiently large net internal magnetic field, $B_{\text {int }}(3)$, to align their net magnetic moment probably along the $\mathrm{Ni}$ magnetic hard axis. It should be noted that in order to extend the $\left(B_{\text {res }, 3}-B_{\text {res }}{ }^{0}\right)$ data down to $117 \mathrm{~K}, B_{\text {res }}(3)$ is taken as $\sim 0.91 \mathrm{~T}$ in the $117-200 \mathrm{~K}$ range because the position of the resonance line $\mathbf{3}$ is not shifted below $\sim 200 \mathrm{~K}$ ( $c f$. Fig. 3(b)).

As mentioned earlier, for a ferromagnetic particle, besides the magnetocrystalline $\left(K_{1}\right)$ anisotropy field and dipolar fields from inter-particle interactions, other effects such as (a) strain and surface chemical effects (e.g., at the $\mathrm{Ni}$ and $\mathrm{MgO}$ interface), (b) shape anisotropy, and (c) spin exchange anisotropy arising from differences in the core vs. surface spins may also contribute to the net internal fields. ${ }^{20}$ For $\mathrm{MgO}: \mathrm{Ni}$ sample, demagnetization effects from the sample's rectangular shape may also contribute to $B_{\text {int }}(i)(i=\mathbf{1}$, 2, 3). Since most of these anisotropy contributions are expected to have complex temperature dependencies, ${ }^{20}$ further in-depth analysis of $\left|B_{\text {res }}-B_{\text {res }}{ }^{0}\right|$ vs. $T$ for $\mathrm{MgO}: \mathrm{Ni}$ cannot be carried out at this time.

\section{Angular dependence of X-band FMR spectra}

A macroscopic alignment of $\mathrm{Ni}$ nanocubes with respect to the $\mathrm{MgO}$ host crystal matrix allowed for an examination of X-band FMR spectra as a function of the crystal orientation angle, $\theta$, with respect to the direction of the external magnetic field $B_{\text {ext }}$. Measurements at $295 \mathrm{~K}$ and $466 \mathrm{~K}$ were carried out by rotating the sample with $\overrightarrow{B_{\text {ext }}}$ in the "out-ofplane" and "in-plane" in $2^{\circ}$ increment.

We define the "out-of-plane" as a rotation along the $[010]_{\mathrm{MgO}}$ direction ( $c f$. Fig. $1(\mathrm{~b})$ ) i.e., $\theta=0^{\circ}$ corresponds to $[100]_{\mathrm{MgO}}$ direction and $\theta=90^{\circ}$ corresponds to $[001]_{\mathrm{MgO}}$ direction. "In-plane" is defined as a rotation along the $[001]_{\mathrm{MgO}}$ direction where $\theta=0^{\circ}$ and $90^{\circ}$, respectively,

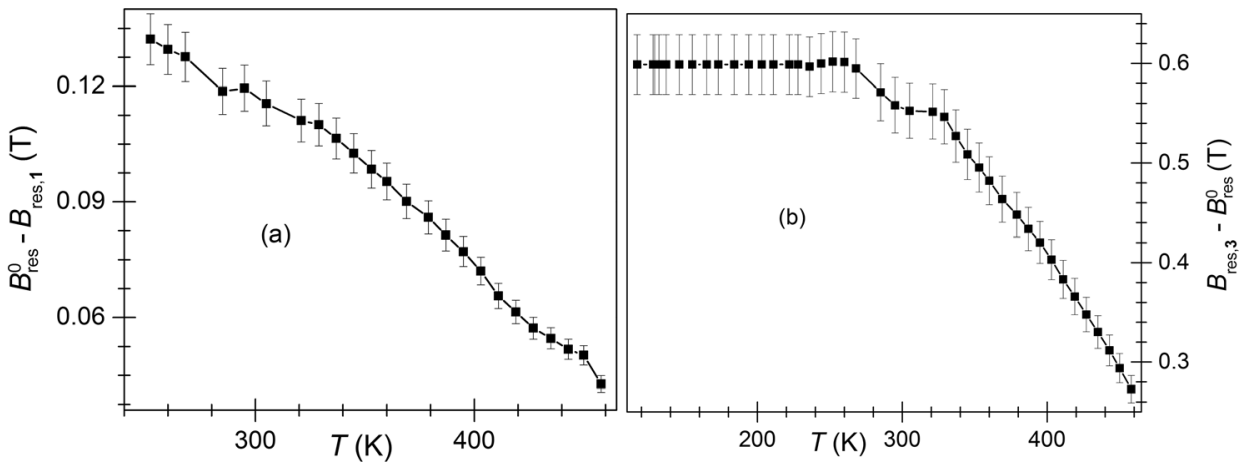

FIG. 6. A difference between the observed FMR resonance position, $B_{\text {res, }}$, and the expected "superparamagnetic" resonance position, $B_{\text {res }}{ }^{0}$, plotted as a function of temperature for the set $\mathbf{1}$ (a) and set 3 (b) Ni nanocubes. The solid lines connecting the experimental data points are provided as a guide to the eye. 

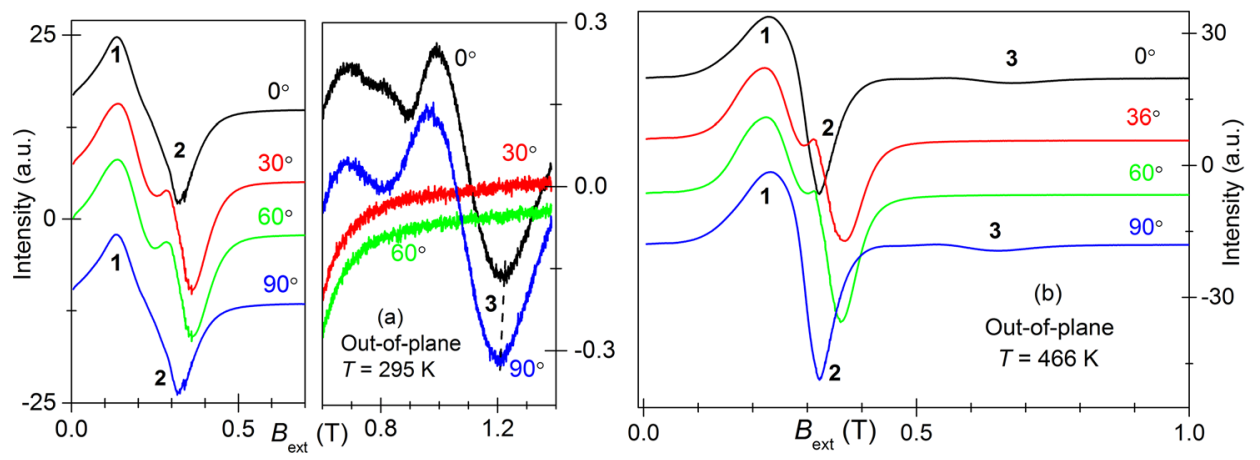

FIG. 7. Experimental X-band ( $9.5 \mathrm{GHz})$ FMR spectra of MgO:Ni for representative $\theta$ angles between $B_{\text {ext }}$ and [100 $]_{\mathrm{MgO}}$ with the $B_{\mathrm{ext}}$ vector being in the crystal $\{010\}$ plane. (a) Room temperature spectra with the high field region magnified in the right panel. (b) High temperature $(T=466 \mathrm{~K})$ spectra. An observation of the resonance lines merging at $\sim 36^{\circ}$ (instead of $30^{\circ}$ ) is most likely due to a slight slipping of the quartz sample rod and/or the goniometer error. At both temperatures, the $\theta=0^{\circ}$ and $90^{\circ}$ orientations correspond to the $B_{\mathrm{ext}} \|[100]_{\mathrm{MgO}}$ and $B_{\mathrm{ext}} \|[001]_{\mathrm{MgO}}$ crystal directions, respectively (see Fig. 1(b)). All spectra are shifted vertically for clarity. See text for further details.

correspond to the $[100]_{\mathrm{MgO}}$ and $[010]_{\mathrm{MgO}}$ crystal directions. At a given temperature, FMR spectra showed a clear $90^{\circ}$ periodicity (see Fig. 7) that was identical for both sample rotation planes. These observations are consistent with the fact that both $\mathrm{MgO}$ and $\mathrm{Ni}$ have a cubic crystal symmetry. At $295 \mathrm{~K}(466 \mathrm{~K})$ when $\theta$ is between $28^{\circ}\left(36^{\circ}\right)$ and $62^{\circ}$, only two resonance lines are observed and, therefore, they could not be unambiguously assigned to a specific $\mathrm{Ni}$ nanocube set $(1,2$, or 3$)$.

At both temperatures and for the out-of-plane and inplane sample rotations, $B_{\text {res }}$ and $\delta B$ of set $\mathbf{1} \mathrm{Ni}$ nanocubes change only by $\sim 10 \mathrm{mT}-20 \mathrm{mT}$ when $\theta$ changes from $0^{\circ}-26^{\circ}$. Under the same conditions, $B_{\text {res }}$ and $\delta B$ of set $2 \mathrm{Ni}$ nanocubes weakly vary by $\sim 30 \mathrm{mT}-40 \mathrm{mT}$. This observation suggests that the set $\mathbf{1}$ and set $\mathbf{2}$ most likely contain Ni nanocubes with a relatively narrow distribution of the internal magnetic field directions.

In contrast, the FMR line of set $\mathbf{3} \mathrm{Ni}$ nanocubes shows a dramatic dependence on $\theta$ under the same conditions. For set 3 nanocubes, at room temperature, while $B_{\text {res }}(\theta)$ varies by $\sim 700 \mathrm{mT}, \delta B(\theta)$ changes by $\sim 270 \mathrm{mT}$ ( $c f$. Figure 8 ), suggesting that set $\mathbf{3}$ contains relatively small $\mathrm{Ni}$ nanocubes with a broad distribution of the directions of the internal magnetic fields. Since $\left|B_{\text {res }}\left(90^{\circ}\right)-B_{\text {res }}\left(0^{\circ}\right)\right|$ in ferromagnetic nanocubes is a measure of the magnitude of the internal magnetic field, $B_{\text {int }}$, we estimate $B_{\text {int }}(\mathbf{3})$ as $\sim 700 \mathrm{mT}$ for set $\mathbf{3}$ nickel nanocubes. We note that the exact composition of set $\mathbf{3}$ nanocubes in $\mathrm{MgO}: \mathrm{Ni}$ is unknown in terms of size, shape, blocking temperature, and the nanocube orientation with respect to the $\mathrm{MgO}$ axes. However, $B_{\text {int }}$ can be still estimated if we neglect the effects of the shape and size and assume that the magnetocrystalline anisotropy is the dominant contributor. Indeed, for a spherical particle composed of a material with cubic anisotropy, $B_{\text {int }}=K_{1} / 4 M_{\mathrm{s}}$, where $K_{1}$ is the magnetocrystalline anisotropy constant and $M_{\mathrm{s}}$ is the saturation magnetization. ${ }^{23}$ At $T=298 \mathrm{~K}, K_{1}=4.5 \times 10^{4} \mathrm{erg} / \mathrm{cm}^{3}$ (bulk nickel) ${ }^{21}$ and $M_{\mathrm{s}}=11.80 \mathrm{emu} / \mathrm{cm}^{3}$ in MgO:Ni. ${ }^{7}$ These values yield $B_{\text {int }}(3) \approx 95.3 \mathrm{mT}$, which is about an order of magnitude smaller than the observed value from FMR. This estimate shows that the magnetocrystalline anisotropy alone is insufficient for explaining the observed values of $B_{\text {int }}(\mathbf{3})$. Thus, other factors, including surface magnetization and the particle shape in particular, should also be taken into account. Nevertheless, we would like to reemphasize that the observation of four maxima in $B_{\text {res, } 3}(\theta)$ is consistent with the expected behavior of nanoparticles exhibiting cubic magnetocrystalline anisotropy.

As mentioned earlier, an increase in temperature increases the thermal fluctuations that reduce the effect of the net internal fields on the FMR line position and the linewidth. Therefore, a variation of $B_{\text {res }}$ and $\delta B$ as a function of the sample orientation in the external magnetic field is expected to be moderate at high temperatures. Indeed, at $466 \mathrm{~K}$ while $B_{\text {res }}(\theta)$ varies by $\sim 300 \mathrm{mT}$ for set $3 \mathrm{Ni}$ nanocubes, $\delta B(\theta)$ for them varies by $\sim 120 \mathrm{mT}$. These values are $\sim 44 \%$ lower than the ones obtained at room temperature.

\section{Frequency dependence of the FMR spectra}

In order to further understand the magnetic nature of the $\mathrm{Ni}$ nanocubes responsible for the resonance lines $\mathbf{1}, \mathbf{2}$, and $\mathbf{3}$ in the FMR spectra of $\mathrm{MgO}: \mathrm{Ni}$, the X-band measurements were expanded to a broader range of magnetic fields and resonant frequencies, $\nu$, from $24 \mathrm{GHz}$ to $330 \mathrm{GHz}$. The measurements were carried out at room temperature with $B_{\text {ext }}$ directed along the $[100]_{\mathrm{MgO}},[010]_{\mathrm{MgO}}$, and $[001]_{\mathrm{MgO}}$ crystal
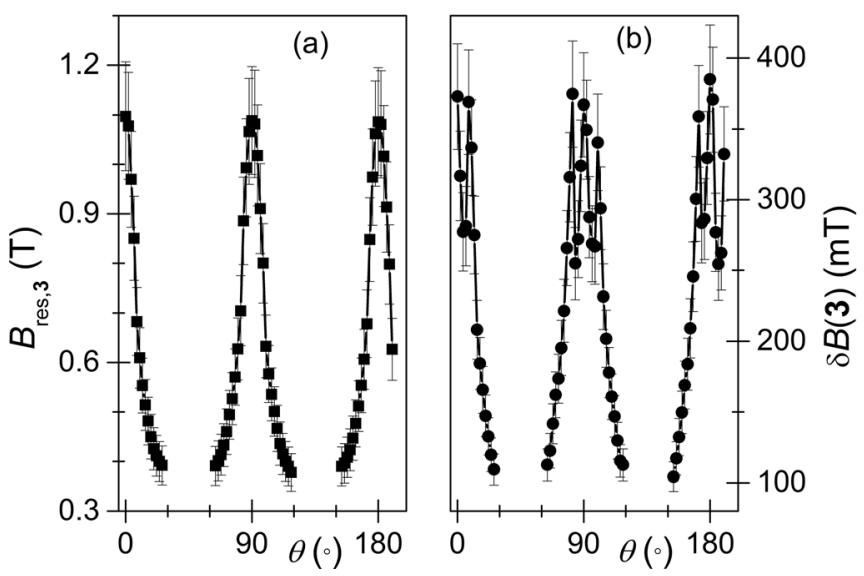

FIG. 8. Angular dependence of (a) $B_{\text {res }}$ and (b) $\delta B$ for set 3 nickel nanocubes obtained from least-squares fitting of X-band $(9.5 \mathrm{GHz})$ FMR spectra for the out-of-plane sample rotation at $295 \mathrm{~K}$. Assignments of the crystal alignment angles are $0^{\circ} \equiv[100]_{\mathrm{MgO}}$ and $90^{\circ} \equiv[001]_{\mathrm{MgO}}$. The solid lines serve as a guide to the eye. 
directions, and as expected, the spectra were identical for all three directions.

Figure 9 displays the FMR spectra at representative resonant frequencies for $B_{\text {ext }} \|[100]_{\mathrm{MgO}}$. As the frequency is increased from $9.5 \mathrm{GHz}$ to $330 \mathrm{GHz}$, the following changes in FMR spectra were observed:

(i) Only two lines at $\sim 0.8 \mathrm{~T}$ and at $\sim 1.3 \mathrm{~T}$ are detected at $24 \mathrm{GHz}$. Interestingly, the shift in the $g$-factor of the resonance line $\mathbf{3}$, defined as $\Delta g(\mathbf{3})=g_{\text {obs }}(\mathbf{3})-g_{\mathrm{Ni}}$ $\approx 1.3$, at $24 \mathrm{GHz}$ and $295 \mathrm{~K}$ is very similar to $\Delta g(\mathbf{3})$ $\approx 1.2$ observed at $9.5 \mathrm{GHz}$ but at a much higher temperature of $\sim 460 \mathrm{~K}$. The observation of the two lines implies that while the Zeeman field corresponding to $24 \mathrm{GHz}$ is sufficient to "saturate" the Ni nanocubes responsible for the resonance line $\mathbf{1}$, it is still not high enough to overcome the internal magnetic field in the set $3 \mathrm{Ni}$ nanocubes that has pinned the magnetization along the hard axis.

(ii) For $\nu>24 \mathrm{GHz}$, only one broad line $(\delta B \sim 0.1-0.3 \mathrm{~T})$ with an asymmetric shape is observed, implying that all the Ni nanocubes in $\mathrm{MgO}: \mathrm{Ni}$ are saturated at these high magnetic fields. The large asymmetric line is likely to be attributed to a broad size distribution of $\mathrm{Ni}$ nanocubes formed in the $\mathrm{MgO}: \mathrm{Ni}$ crystal.

(iii) Crystal rotation at $240 \mathrm{GHz}$ (data not shown) reveals virtually no angular dependence of the resonance position of the broad asymmetric line that stayed at $\mathrm{g} \approx 2.2$ implying that at these magnetic fields, the resonance line is fairly isotropic in nature i.e., all the effects of the internal magnetic fields become negligible, and the magnetic moment vectors of the individual $\mathrm{Ni}$ nanocubes are all pointing along the direction of the external magnetic field.

For all the FMR spectra above $24 \mathrm{GHz}$, an additional two component narrow line $(\delta B \sim 20 \mathrm{mT})$ overlapping the broad FMR line is also observed. As the FMR resonance broadens with increasing frequency, the two-component line also becomes more distinct as the frequency increases. Also, its $g$-factor and the line width remain almost unchanged over the entire microwave frequency range accessible experimentally.
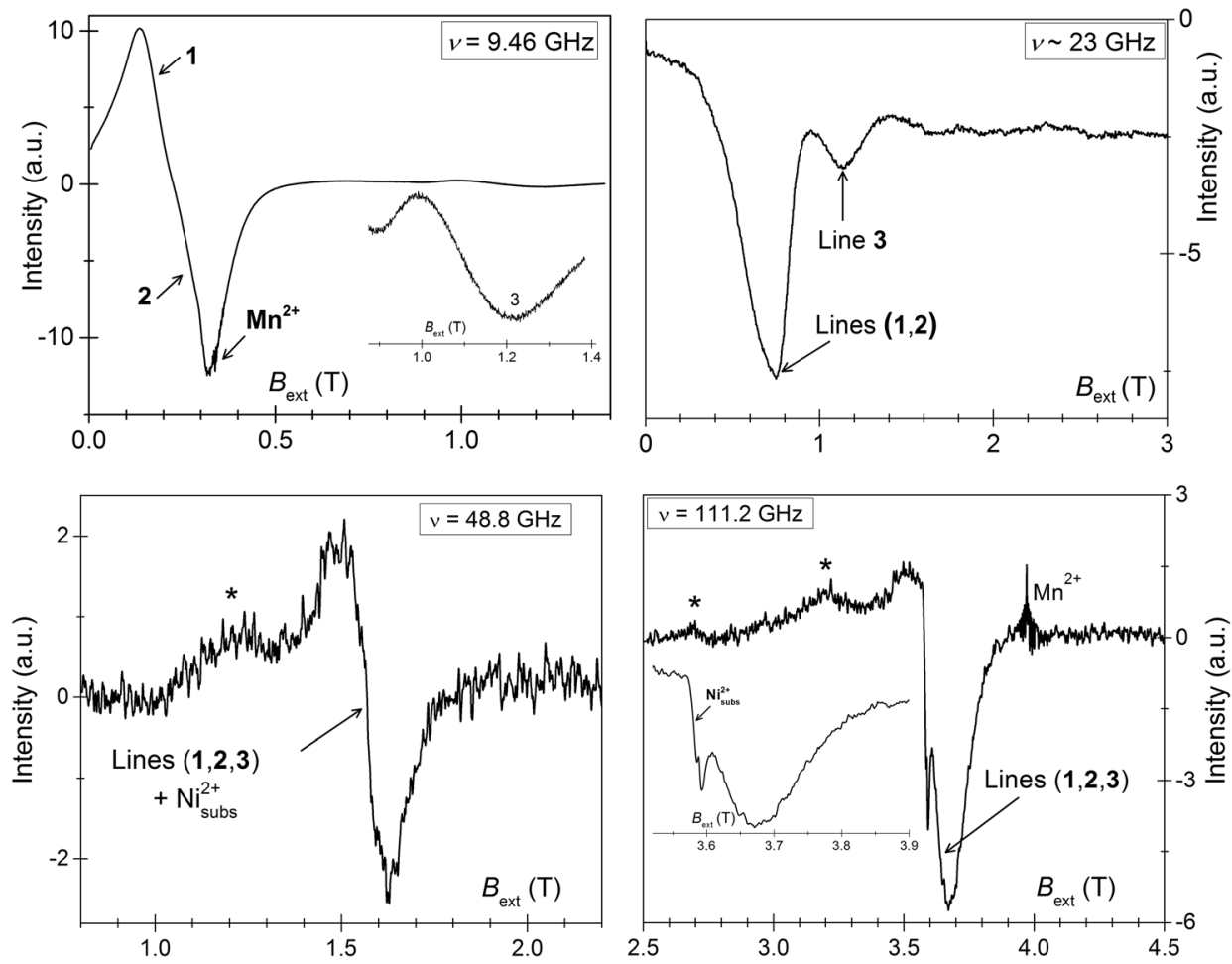

FIG. 9. Representative multi-frequency FMR spectra of MgO:Ni obtained at $300 \mathrm{~K}$ with the external magnetic field oriented along the $[100]_{\mathrm{MgO}}$ crystal direction. Lines marked by "*" are impurity and/or signals attributed to molecular oxygen. $24 \mathrm{GHz}$ FMR spectrum is recorded in the absorption mode. Insets for $v>50 \mathrm{GHz}$ are zoomin of the spectral components assigned to a substitutional $\mathrm{Ni}^{2+}(g \sim 2.2)$ signal overlapping the broad line originating from Ni nanocubes in sets $\mathbf{1}, \mathbf{2}$, and $\mathbf{3}$. See text for further details.

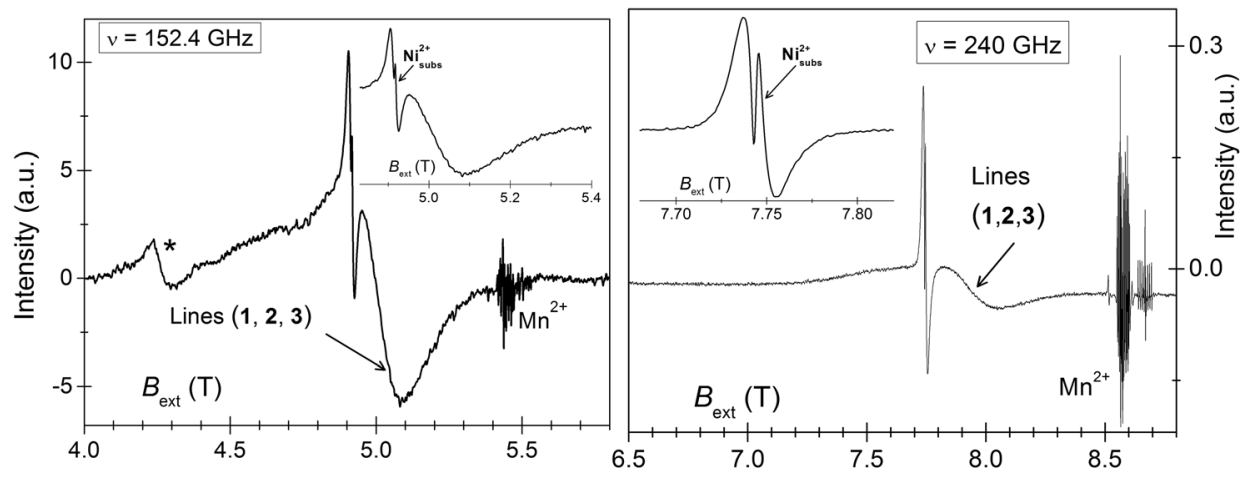


A striking feature of the sharp line is its two components with one component having an inverted phase with respect to the other component. Based on our electron magnetic resonance spectroscopic studies ${ }^{24}$ of paramagnetic nickel doped $\mathrm{MgO}$ single crystal where $\mathrm{Ni}$ is exclusively present as a substitutional dopant, we assign this line to a small quantity of isolated $\mathrm{Ni}^{2+}$ ions ( $\operatorname{spin} s=1$ ) present in the substitutional sites. The inverted phase of one component with respect to other component is due to the known cross-relaxation phenomenon $^{25-27}$ that persists up to the highest microwave frequency studied in this work. Finally, based on a comparison of the double integrated areas of the narrow line and the broad FMR line, we estimate the percentage of the substitutional $\mathrm{Ni}^{2+}$ in $\mathrm{MgO}: \mathrm{Ni}$ as $\sim 1.6 \%$. Considering that $\sim 0.5$ at. wt. $\%$ of $\mathrm{Ni}$ is used in the synthesis of $\mathrm{MgO}: \mathrm{Ni}$, percentage of the substitutional $\mathrm{Ni}^{2+}$ in $\mathrm{MgO}: \mathrm{Ni}$ is $\sim 0.008$ at. wt. $\%$. These results establish the superior sensitivity of the electron magnetic resonance spectroscopy in identifying different types of magnetic species in a heterogeneous system such as $\mathrm{MgO}: \mathrm{Ni}$.

Figure 10 displays the resonance field as a function of microwave frequency for the $\mathrm{Ni}$ nanocube sets $\mathbf{1}$ and $\mathbf{3}$. According to the resonance condition $B_{\text {res }}=B^{0}{ }_{\text {res }}=(h / g \beta) \nu$, a linear regression fit of the high-frequency data $(50 \mathrm{GHz}$ $-330 \mathrm{GHz}$ ) yields $g=2.198$, which is a characteristic value of $\mathrm{Ni}$ metal ions. In order to obtain the magnitude of the internal magnetic fields, $B_{\text {res, } 3}$, data in the $9.5 \mathrm{GHz}-50 \mathrm{GHz}$ frequency range were fitted to $B_{\text {res }}=(h / g \beta) \nu+B_{\text {int }}$ with $B_{\text {int }}(3)=1.03 \mathrm{~T}$ and $g_{\text {eff }}(3)=6.44$. Such a large $B_{\text {int }}(3)$ is consistent with an exceptional sensitivity of the position and the linewidth of line $\mathbf{3}$ resonance with respect to the direction of the external magnetic field observed at the X-band. Unfortunately, relatively poor quality of multi-frequency FMR spectra prevented us from plotting the linewidth $v$ s. the frequency.

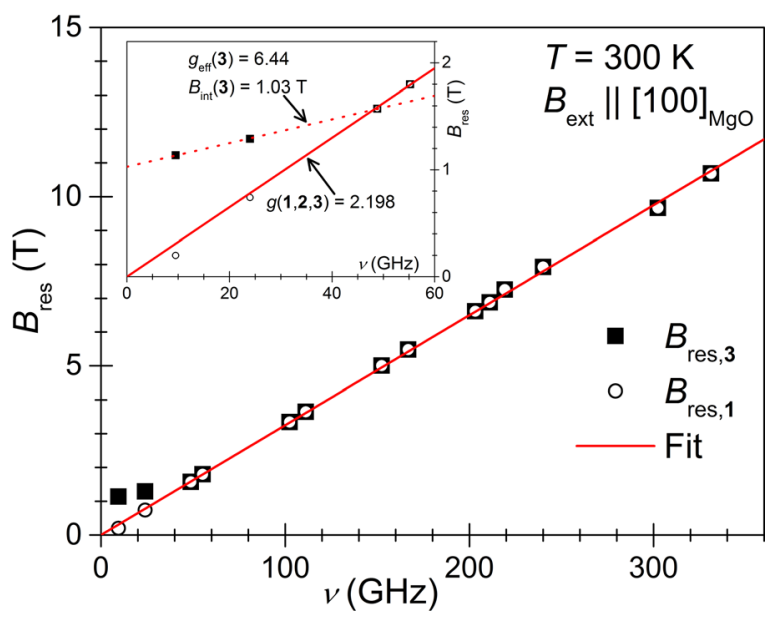

FIG. 10. Resonance field as a function of frequency for the set $\mathbf{1}$ and set $\mathbf{3}$ $\mathrm{Ni}$ nanocubes in MgO:Ni. External magnetic field is oriented along the $[100]_{\mathrm{MgO}}$ crystal direction. The solid red line represents a linear regression of high-frequency $(\nu>24 \mathrm{GHz})$ data to $h \nu=g \beta B^{0}{ }_{\text {res. }} g$-factor from the slope $(=0.0325)$ is calculated as 2.198 , which is a characteristic value for Ni metal ions. The inset shows a deviation of the low-frequency data $(\nu \leq 24 \mathrm{GHz})$ from the straight line, where the dotted line represents the linear regression of the set $\mathbf{3}$ Ni nanocubes' low-frequency data with the values $g_{\text {eff }}(\mathbf{3})=6.44$ and $B_{\text {int }}(\mathbf{3})=1.03 \mathrm{~T}$.

\section{CONCLUSIONS}

The X-band FMR spectrum of nickel nanocubes grown epitaxially in a diamagnetic magnesium oxide lattice was found to feature predominantly three resonance lines $(\mathbf{1}, \mathbf{2}$, and 3) spread over the entire experimentally accessible ranges of the external magnetic field. These lines were assigned based on the observed temperature and crystal orientation dependencies of the spectral parameters. Specifically, the low-field resonance line (line $\mathbf{1}, g_{\text {eff }} \sim 3.6$ ) was assigned to large multidomain nickel nanocubes, while the line $2\left(g_{\text {eff }} \sim 2.2\right)$ was assigned to magnetically saturated nickel nanocubes. The high-field signal (line $\mathbf{3}, g_{\text {eff }} \sim 6.2$ ) was tentatively assigned to small nickel nanocubes with their hard magnetization axis oriented along or close to the external magnetic field direction.

Increasing the external magnetic fields and the corresponding microwave frequency from $9.5 \mathrm{GHz}$ to $24 \mathrm{GHz}$ causes the low-field FMR signals (line $\mathbf{1}$ and 2) to merge into a single, fairly symmetric line centered at $\sim 0.78 \mathrm{~T}(\mathrm{~g} \approx 2.2)$ corresponding to the "superparamagnetic" $\mathrm{Ni}$ species. Surprisingly, for set $\mathbf{3} \mathrm{Ni}$ nanocubes, the internal magnetic field, arising from various anisotropy contributions, still persists at $24 \mathrm{GHz}$. This observation suggests that the internal magnetic field in the set $\mathbf{3}$ nickel nanocubes is at least of the order of $24 \mathrm{GHz}(\sim 1.25 \mathrm{~T})$. A further increase in the external magnetic field and the resonant frequency up to $330 \mathrm{GHz}$ resulted in a single broad asymmetric FMR line overlapping with a sharp two-component signal. The latter twocomponent signal is attributed to a minute amount of $(\sim 0.008$ at. wt. $\%)$ substitutional $\mathrm{Ni}^{2+}$ ions. While we were able to estimate semi-quantitatively the net internal field in set 3 nickel nanocubes as $\sim 1 \mathrm{~T}$ from the $B_{\text {res }}$ vs. the $v$ plot, the complex size and anisotropy axes distribution along with sizable contributions of magnetocrystalline, size, and shape anisotropies to the internal magnetic field prevented us from modeling the temperature and the angular dependence data collected at the X-band. Nonetheless, assuming nickel nanocubes in $\mathrm{MgO}: \mathrm{Ni}$ retain the magnetization axes of the bulk nickel, we believe that set $\mathbf{3}$ nickel nanocubes are small sized with their internal field oriented along the hard magnetization axis of $\langle 100\rangle_{\mathrm{Ni}}$. Further, an observation of multiple FMR lines with large linewidths agrees very well with the bulk magnetic susceptibility studies, ${ }^{5,7}$ which indicated a very broad distribution of blocking temperatures consistent with a broad nanocube size distribution. More importantly, remarkable sensitivity of the FMR line position and linewidth to temperature, sample orientation, and microwave frequency allowed us to distinguish between the three major types of nickel nanocubes in MgO:Ni. In conclusion, FMR studies of magnetic nanoparticles and arrays could provide additional information on local anisotropic magnetic fields and complement the conventional bulk magnetic susceptibility studies that provide only the average physical picture of various magnetic environments of these anisotropic nanostructures embedded in a diamagnetic host matrix.

\section{ACKNOWLEDGMENTS}

EMR instrumentation at NCSU is supported by Grant Nos. NIH S10RR023614, NSF CHE-0840501, and NCBC 
2009-IDG-1015. S. N. and A. I. S. are grateful to the U.S. Department of Energy's Office of Basic Energy Sciences for financial support (Grant No. DE-FG02-02ER15354) to the experimental FMR studies and data interpretation. S. N. thanks Dr. Alexey Kovalev and Dr. Stephen Hill for help with K-band $(24 \mathrm{GHz})$ measurements and helpful discussions. S. N. gratefully acknowledges the National High Magnetic Field Laboratory (NHMFL) for high frequency/high field EMR measurements and Dr. Johan van Tol for helpful discussions. NHMFL is funded by the NSF through a Cooperative Agreement DMR 1157490 and the State of Florida.

${ }^{1}$ K. M. Krishnan, A. B. Pakhomov, Y. Bao, P. Blomqvist, Y. Chun, M. Gonzales, K. Griffin, X. Ji, and B. K. Roberts, J. Mater. Sci. 41, 793 (2006).

${ }^{2}$ H.-W. Zhang, Y. Liu, and S.-H. Sun, Front. Phys. China 5, 347 (2010).

${ }^{3}$ H. Zhou and J. Narayan, J. Nanopart. Res. 8, 595 (2006).

${ }^{4}$ J. Narayan, Y. Chen, and R. M. Moon, Phys. Rev. Lett. 46, 1491 (1981).

${ }^{5}$ S. Ramachandran, J. Narayan, and J. T. Prater, Appl. Phys. Lett. 90, 132511 (2007).

${ }^{6}$ R. Freer, J. Mater. Sci. 15, 803 (1980).

${ }^{7}$ J. Narayan, S. Nori, S. Ramachandran, and J. T. Prater, JOM 61, 76 (2009).

${ }^{8}$ M. Chipara, R. Skomski, R. Kirby, and D. J. Sellmyer, J. Mater. Res. 26, 2169 (2011).
${ }^{9}$ K. Seemann, H. Leiste, and Ch. Klever, J. Magn. Magn. Mater. 322, 2979 (2010)

${ }^{10}$ R. J. Usselman, S. E. Russek, M. T. Klem, M. A. Allen, T. Douglas, M. Young, Y. U. Idzerda, and D. J. Singel, J. Appl. Phys. 112, 084701 (2012).

${ }^{11}$ M. M. Can, M. Coskun, and T. Firat, J. Nanopart. Res. 13, 5497 (2011).

${ }^{12}$ J. Lindner, C. A. Hassel, V. Trunova, F. M. Römer, S. Stienen, and I. Barsukov, J. Nanosci. Nanotechnol. 10, 6161 (2010).

${ }^{13}$ C. Carbone, F. Di Benedetto, C. Sangregorio, P. Marescotti, L. A. Pardi, and L. Sorace, J. Phys. Chem. C 112, 9988 (2008).

${ }^{14}$ A. O. Ankiewicz, J. S. Martins, M. C. Carmo, M. Grundmann, S. Zhou, H. Schmidt, and N. A. Sobolev, J. Appl. Phys. 107, 09B518 (2010).

${ }^{15}$ R. V. Upadhyay, K. Parekh, and R. V. Mehta, Phys. Rev. B 68, 224434 (2003).

${ }^{16}$ M. Mola, S. Hill, P. Goy, and M. Gross, Rev. Sci. Instrum. 71, 186 (2000).

${ }^{17}$ A. K. Hassan, L. A. Pardi, J. Krzystek, A. Sienkiewicz, P. Goy, M. Rohrer, and L.-C. Brunel, J. Magn. Res. 142, 300 (2000).

${ }^{18}$ J. van Tol, L.-C. Brunel, and R. J. Wylde, Rev. Sci. Instrum. 76, 074101 (2005).

${ }^{19}$ EPRcalc program was developed by Dr. Johan van Tol at the National High Magnetic Field Laboratory, Tallahassee, FL.

${ }^{20}$ S. Bedanta and W. Kleemann, J. Phys. D: Appl. Phys. 42, 013001 (2009).

${ }^{21}$ J. Crangle and G. M. Goodman, Proc. R. Soc. London A 321, 477 (1971).

${ }^{22}$ C. A. Neugebauer, Phys. Rev. 116, 1441 (1959).

${ }^{23} \mathrm{H}$. Li, M. T. Klem, K. B. Sebby, D. J. Singel, M. Young, T. Douglas, and Y. U. Idzerda, J. Magn. Magn. Mater. 321, 175 (2009).

${ }^{24}$ J. Narayan, S. Nori, D. K. Pandya, D. K. Avasthi, and A. I. Smirnov, Appl. Phys. Lett. 93, 082507 (2008).

${ }^{25}$ J. W. Orton, P. Auzins, and J. E. Wertz, Phys. Rev. Lett. 4, 128 (1960).

${ }^{26}$ J. W. Orton, P. Auzins, and J. E. Wertz, Phys. Rev. 119, 1691 (1960).

${ }^{27}$ S. R. P. Smith, F. Dravnieks, and J. E. Wertz, Phys. Rev. 178, 471 (1969). 